\title{
Borboletas (Lepidoptera: Papilionoidea e Hesperioidea) do Sudoeste do Pampa Brasileiro, Uruguaiana, Rio Grande do Sul, Brasil
}

\author{
Pamela Laiz Paré da Rosa ${ }^{1,3}$, Enrique Querol Chiva ${ }^{1}$ \& Cristiano Agra Iserhard ${ }^{2}$ \\ ${ }^{1}$ Curso de Ciências Biológicas, Pontifícia Universidade Católica do Rio Grande do Sul-PUCRS, \\ Campus Uruguaiana, BR 472, Km 7 \\ ${ }^{2}$ Programa de Pós-graduação em Biologia Animal, Departamento de Zoologia, \\ Instituto de Biociências, Universidade Federal do Rio Grande do Sul-UFRGS, \\ Av. Bento Gonçalves, n. 9500, CEP 91501-970, Porto Alegre, RS, Brasil \\ ${ }^{3}$ Autor para correspondência: Pamela Laiz Paré da Rosa, e-mail: pamela_pare@ hotmail.com
}

ROSA, P.L.P., CHIVA, E.Q. \& ISERHARD, C.A. Butterflies (Lepidoptera: Papilionoidea and Hesperioidea) in the Southwestern Brazilian Grasslands, Uruguaiana, Rio Grande do Sul State, Brazil. Biota Neotrop. 11(1): http://www.biotaneotropica.org.br/v11n1/en/abstract?inventory+bn00411012011.

\begin{abstract}
Pampa" is the Brazilian biome that occurs in only one state. Besides grasslands formation, this biome has a variety of other ecosystems This work aimed to inventory the butterflies of the Parque Natural Municipal de Uruguaiana, contributing to the knowledge of this group of insects in this region. Three transects were covered in each sampling occasion: riparian forest (MC), margin of Uruguay river (MR) and grasslands (CS). After four samples along one year and a total of 99 net-hours of sampling effort 46 species were recorded, including two new records for Rio Grande do Sul and one new record for Brazil.
\end{abstract}

Keywords: Brazilian Pampa, conservation, riparian forest, Southern Brazil, species richness.

ROSA, P.L.P., CHIVA, E.Q. \& ISERHARD, C.A. Borboletas (Lepidoptera: Papilionoidea e Hesperioidea) do Sudoeste do Pampa Brasileiro, Uruguaiana, Rio Grande do Sul, Brasil. Biota Neotrop. 11(1): http://www. biotaneotropica.org.br/v11n1/pt/abstract?inventory+bn00411012011.

Resumo: O Pampa é o único bioma Brasileiro que ocorre em apenas um estado. Além de formações campestres, possui uma variedade de ecossistemas associados. Este trabalho tem como objetivo realizar um inventário das borboletas do Parque Natural Municipal de Uruguaiana, contribuindo com o conhecimento deste grupo de insetos desta região. Foram percorridas três transecções em cada ocasião amostral: Mata Ciliar (MC), Margem do Rio Uruguai (MR), e Campo Sujo (CS). Após quatro amostragens ao longo de um ano e um total de 99 horas-rede de esforço amostral foram registradas 46 espécies, ocorrendo dois registros novos para o Rio Grande do Sul e um registro novo para o Brasil.

Palavras-chave: Pampa, conservação, mata ciliar, Sul do Brasil, riqueza de espécies. 


\section{Introdução}

O Pampa é o único bioma brasileiro que ocorre em apenas um estado (Paz et al. 2008), correspondendo a 2,07\% do território Nacional e 63\% do território do Rio Grande do Sul (Instituto... 2005), constituindo-se em ecossistemas naturais com alta diversidade de espécies vegetais e animais (Pillar et al. 2009). Este bioma abriga outros ecossistemas protegidos por lei, como banhados, áreas alagadas e matas ciliares que servem de refúgio para a fauna (Bortoluzzi \& Souza 2007). A sua conservação tem sido ameaçada pela conversão em culturas anuais de soja e arroz, da silvicultura de árvores exóticas destinadas a produção de celulose para exportação e pela degradação associada à invasão de espécies exóticas (Pillar et al. 2009).

Nas últimas décadas cerca da metade da superfície originalmente coberta com os Campos Sulinos no Rio Grande do Sul foi transformada em outros tipos de cobertura vegetal (Overbeck et al. 2007). Além disso, o Pampa está pobremente representado no sistema de áreas protegidas, atualmente apenas $453 \mathrm{~km}^{2}$ estão contemplados em Unidades de Conservação de proteção integral, o que equivale a menos de $0,5 \%$ da área total desta formação vegetal (Pillar et al. 2009). Isto denota quanta informação e biodiversidade tem sido perdida e a negligência acerca de projetos de monitoramento e conservação destas áreas nativas de campo.

O conhecimento sobre a riqueza e composição de invertebrados terrestres do Pampa é extremamente precário e com grandes lacunas, de acordo com avaliação realizada para a definição do status do conhecimento da biodiversidade brasileira (Lewinsohn 2006). Com a fauna de borboletas este cenário não é muito diferente, são escassas as informações acerca deste táxon nos diferentes ambientes deste Bioma. A disponibilização de dados relativos à riqueza, distribuição e composição de espécies é fundamental para avaliação e monitoramento de borboletas (Emery et al. 2006). As borboletas são sensíveis a mudanças ambientais, o que as conferem a possibilidade de serem utilizadas como bioindicadoras (Brown \& Freitas 1999).

Estudos de diversidade de borboletas têm aumentado nas duas últimas décadas, contribuindo a América Latina sobremaneira neste novo panorama (Lamas 2008). No Rio Grande do Sul, pesquisas relativas a inventários e estudos de diversidade de borboletas vem sendo desenvolvidos com maior frequência, pelo menos, nos últimos dez anos e vem aumentando gradativamente (Morais et al. 2007). Em relação ao Pampa, trabalhos históricos foram realizados na região sudeste e missioneira do Rio Grande do Sul por Biezanko (1949, 1958, 1959a, b, 1960a, b, c, d, e, 1963). Posteriormente, tais trabalhos foram revisados e novas amostragens foram realizadas no trabalho de Krüger \& Silva (2003). Paz et al. (2008) realizaram um inventário das borboletas da superfamília Papilionoidea (com exceção de Lycaenidae) em duas regiões da Serra do Sudeste, em áreas de campo e mata ciliar nos municípios de Canguçu e Caçapava do Sul, registrando 81 espécies de Nymphalidae, Pieridae e Papilionidae. As borboletas da formação vegetacional Savana Estépica do Parque Estadual do Espinilho (PEE) e entorno, localizado no município de Barra do Quaraí, foram estudadas por Marchiori \& Romanowski (2006). Foram registradas 97 espécies de borboletas e destas, 10 novos registros para o estado, o que demonstra a riqueza e a necessidade de mais estudos focados no Pampa.

Os inventários de borboletas supracitados com esforço amostral padronizado realizados nos Campos Sulinos (Pampa e Campos de Cima da Serra) do Rio Grande do Sul evidenciaram a estrita associação de determinados grupos de borboletas com ambientes campestres nativos, podendo tais grupos ser potenciais indicadores de campos preservados (Morais et al. 2007, Romanowski et al. 2009, Iserhard et al. 2010).
Este trabalho tem como objetivo realizar um inventário rápido das borboletas de diferentes ambientes do Parque Natural Municipal de Uruguaiana, contribuindo com o conhecimento deste grupo de insetos na região do extremo sudoeste do Pampa.

\section{Material e Métodos}

\section{1. Área de estudo}

O Parque Natural Municipal de Uruguaiana (PNMU) ( $29^{\circ} 30^{\prime} 05^{\prime}$ 'S e 56 49' 56” W) está localizado na região Sudoeste do Rio Grande do Sul, à margem esquerda do Rio Uruguai, a 50 km ao norte do município de Uruguaiana, na localidade de São Marcos. A formação geológica do Parque é constituída pelas formações Serra Geral e Deserto de Botucatu (Radambrasil 1986) possuindo uma mata ciliar relativamente preservada que tem sofrido ação das atividades de agropecuária, comuns na região. A região encontra-se na classificação de clima subtropical, subúmido com temperatura média anual de 19,7 ${ }^{\circ} \mathrm{C}$ e precipitação anual de $1346 \mathrm{~mm}$ (Maluf 2000).

\section{Amostragem}

Foram realizadas quatro expedições a campo, nas estações de inverno, primavera, verão e outono entre 2008 e 2009. Três trilhas foram selecionadas em ambientes diferentes. Uma das áreas constituise em uma transecção percorrida dentro de uma Mata Ciliar (MC), a qual apresentava um dossel uniforme, com pouca vegetação no estrato primário, e solo coberto de serapilheira. A segunda transecção foi percorrida na Margem do Rio Uruguai (MR), com presença de gramíneas e solo arenoso, havendo lagoas marginais entre a margem e a mata ciliar. No entorno do PNMU estava localizada a transecção de Campo Sujo (CS), circundada pelas atividades de agropecuária.

Cada transecção foi percorrida durante três horas por ocasião amostral com a participação de três amostradores com rede entomológica. $\mathrm{O}$ esforço amostral foi padronizado multiplicando-se o número de horas de amostragem pelo número de amostradores com rede. Todas as amostragens foram realizadas entre as 09:00 e 19:00 (vide Marchiori \& Romanowski 2006, Paz et al. 2008). Durante a primavera, a trilha MR não foi amostrada, decorrente da cheia no Rio Uruguai. Em cada ocasião amostral, amostradores experientes munidos de rede entomológica percorreram as transecções a passo lento, procurando ativamente por borboletas ao longo e ao redor da trilha, visando cobrir um espectro amplo de alturas de vôo. Borboletas avistadas foram capturadas com a rede entomológica. Sempre que possível, a identificação era realizada no local - se necessário, com o auxílio de guias de campo especializados (Canals 2000, 2003) - e, a seguir, a borboleta liberada. Indivíduos distintamente conspícuos, em situações acessíveis e/ou posições marcadamente óbvias que tornassem sua identificação segura possível sem a captura com rede foram amostrados apenas visualmente e registrados em planilha de campo, visando manter a manipulação das borboletas ao mínimo necessário. Quando aplicável, recorreu-se também ao uso de fotografia digital como auxílio para identificação.

Para coletas de exemplares testemunho ou de difícil identificação em campo, ao menos um indivíduo de cada espécie foi capturado para posterior montagem e identificação em laboratório. Os espécimes estão depositados na Coleção de Lepidoptera do Departamento de Zoologia, Universidade Federal do Rio Grande do Sul. Os exemplares foram identificados através de bibliografia especializada (D'Abrera 1981, Canals 2000,2003) e a consulta a especialistas e a nomenclatura está atualizada segundo Lamas (2004) e Mielke (2005).

\section{Análise dos dados}

Para confirmação de novos registros para o estado foram consultados os trabalhos de Weymer (1894), Biezanko (1958, 
Tabela 1. Lista de espécies de borboletas registradas no período de julho de 2008 a maio de 2009, no Parque Natural Municipal de Uruguaiana (PNMU), Uruguaiana, Rio Grande do Sul. (CS) Campo Sujo, (MC) Mata Ciliar, (MR) Margem do rio Uruguai, (S) Riqueza de espécies, (\#) novos registros para o Estado, (*) novo registro para o Brasil.

Table 1. Butterfly species list in Parque Natural Municipal de Uruguaiana (PNMU), recorded between July 2008 and May 2009, Uruguaiana, Rio Grande do Sul State. (CS) Grasslands, (MC) riparian forest, (MR) margin of Uruguay river, (S) species richness, (\#) new records for Rio Grande do Sul, (*) new record for Brazil.

\begin{tabular}{|c|c|c|c|c|}
\hline \multirow[t]{2}{*}{ Famílias/Subfamílias } & \multirow[t]{2}{*}{ Espécies } & \multicolumn{3}{|c|}{ PNMU } \\
\hline & & CS & MC & MR \\
\hline \multicolumn{5}{|l|}{ NYMPHALIDAE $(\mathrm{S}=23)$} \\
\hline \multirow[t]{7}{*}{ Nymphalinae } & Anartia amathea roeselia (Eschscholtz, 1821) & - & $\mathrm{X}$ & - \\
\hline & Anartia jatrophae jatrophae (Linnaeus, 1763) & - & $\mathrm{X}$ & $\mathrm{X}$ \\
\hline & Junonia evarete (Cramer, 1779) & $\mathrm{X}$ & $\mathrm{X}$ & $\mathrm{X}$ \\
\hline & Ortilia ithra (W. F. Kirby, 1900) & $\mathrm{X}$ & $\mathrm{X}$ & $\mathrm{X}$ \\
\hline & Tegosa orobia (Hewitson, 1864) & - & - & $\mathrm{X}$ \\
\hline & Vanessa braziliensis (Moore, 1883) & $\mathrm{X}$ & $\mathrm{X}$ & $\mathrm{X}$ \\
\hline & Vanessa carye (Hübner, 1812) & - & - & $\mathrm{X}$ \\
\hline Biblidinae & Eunica tatila bellaria Fruhstorfer, 1908 & - & - & $\mathrm{X}$ \\
\hline Satyrinae & Paryphthimoides phronius (Godart, 1824) & $\mathrm{X}$ & $\mathrm{X}$ & - \\
\hline \multirow[t]{4}{*}{ Heliconiinae } & Agraulis vanillae maculosa (Stichel, 1908) & $\mathrm{X}$ & - & $\mathrm{X}$ \\
\hline & Dryas iulia alcionea (Cramer, 1779) & - & $\mathrm{X}$ & - \\
\hline & Euptoieta hortensia (Blanchard, 1852) & - & - & $\mathrm{X}$ \\
\hline & Heliconius erato phyllis (Fabricius, 1775) & $\mathrm{X}$ & $\mathrm{X}$ & - \\
\hline Limenitidinae & Adelpha thessalia indefecta Fruhstorfer, 1913 & - & $\mathrm{X}$ & - \\
\hline \multirow[t]{2}{*}{ Ithomiinae } & Dircenna dero celtina Burmeister, 1878 & - & $\mathrm{X}$ & - \\
\hline & Mechanitis lysimnia lysimnia (Fabricius, 1793) & - & $\mathrm{X}$ & - \\
\hline \multirow[t]{2}{*}{ Danainae } & Danaus erippus (Cramer, 1775) & $\mathrm{X}$ & - & $\mathrm{X}$ \\
\hline & Danaus gilippus gilippus (Cramer, 1775) & $\mathrm{X}$ & - & - \\
\hline \multirow[t]{2}{*}{ Charaxinae } & Memphis moruus (Fabricius, 1775) & $\mathrm{X}$ & - & - \\
\hline & Fountainea glycerium cratais (Hewitson, 1874)\# & - & $\mathrm{X}$ & - \\
\hline \multirow[t]{2}{*}{ Apaturinae } & Doxocopa kallina (Staudinger, 1886) & - & $\mathrm{X}$ & $\mathrm{X}$ \\
\hline & Doxocopa laurentia laurentia (Godart, 1824) & - & $\mathrm{X}$ & - \\
\hline Libytheinae & Libytheana carinenta (Cramer, 1777) & - & - & $\mathrm{X}$ \\
\hline \multicolumn{5}{|l|}{ PIERIDAE ( $S=7)$} \\
\hline \multirow[t]{5}{*}{ Coliadinae } & Colias lesbia lesbia (Fabricius, 1775) & $\mathrm{X}$ & - & - \\
\hline & Eurema deva (Doubleday, 1847) & $\mathrm{X}$ & - & $\mathrm{X}$ \\
\hline & Eurema elathea (Cramer, 1777) & $\mathrm{X}$ & $\mathrm{X}$ & $\mathrm{X}$ \\
\hline & Pyrisitia leuce leuce (Boisduval, 1836) & $\mathrm{X}$ & - & - \\
\hline & Pyrisitia nise tenella (Boisduval, 1836) & $\mathrm{X}$ & $\mathrm{X}$ & $\mathrm{X}$ \\
\hline Pierinae & Tatochila mercedis vanvolxemii (Capronnier, 1874)* & $\mathrm{X}$ & - & - \\
\hline Dismorphiinae & Enantia lina psamathe (Fabricius, 1793) & - & $\mathrm{X}$ & $\mathrm{X}$ \\
\hline \multicolumn{5}{|l|}{ PAPILIONIDAE $(\mathrm{S}=1)$} \\
\hline Papilioninae & Mimoides lysithous eupatorion (Lucas, 1859) & $\mathrm{X}$ & - & - \\
\hline \multicolumn{5}{|l|}{ LYCAENIDAE $(\mathrm{S}=2)$} \\
\hline \multirow[t]{2}{*}{ Theclinae } & Atlides polybe (Linnaeus, 1763) & - & $\mathrm{X}$ & - \\
\hline & Pseudolycaena marsyas (Linnaeus, 1758) & $\mathrm{X}$ & $\mathrm{X}$ & - \\
\hline \multicolumn{5}{|l|}{ RIODINIDAE $(\mathrm{S}=2)$} \\
\hline \multirow[t]{2}{*}{ Riodininae } & Aricoris epulus signata (Stichel, 1910) & $\mathrm{X}$ & - & $\mathrm{X}$ \\
\hline & Riodina lysippoides Berg, 1882 & $\mathrm{X}$ & - & - \\
\hline \multicolumn{5}{|l|}{ HESPERIIDAE $(\mathrm{S}=11)$} \\
\hline Pyrrhopyginae & Pyrrhopyge pelota Plötz, 1879 \# & $\mathrm{X}$ & - & - \\
\hline \multirow[t]{3}{*}{ Hesperiinae } & Hylephila phyleus phyleus (Drury, 1773) & $\mathrm{X}$ & - & $\mathrm{X}$ \\
\hline & Nyctelius nyctelius nyctelius (Latreille, 1824) & - & - & $\mathrm{X}$ \\
\hline & Panoquina ocola ocola (W.H. Edwards, 1863) & $\mathrm{X}$ & - & - \\
\hline Pyrginae & Carrhenes canescens pallida Röber, 1925 & $\mathrm{X}$ & - & - \\
\hline & Mylon maimon (Fabricius, 1775) & - & $\mathrm{X}$ & - \\
\hline & Pyrgus orcus (Stoll, 1780) & $\mathrm{X}$ & $\mathrm{X}$ & $\mathrm{X}$ \\
\hline & Pyrgus orcynoides (Giacomelli, 1928) & - & - & $\mathrm{X}$ \\
\hline & Urbanus simplicius (Stoll, 1790) & $\mathrm{X}$ & $\mathrm{X}$ & - \\
\hline & Urbanus teleus (Hübner, 1821) & $\mathrm{X}$ & $\mathrm{X}$ & - \\
\hline & Xenophanes tryxus (Stoll, 1780) & - & $\mathrm{X}$ & - \\
\hline Riqueza de espécies & & 26 & 24 & 21 \\
\hline
\end{tabular}


1959a, b, 1960a, b, c, d, e, 1963), Biezanko \& Mielke (1973), Mielke (1980a, b), Teston \& Corseuil (1998, 2000, 2001, 2008a, b, c), Kruger \& Silva (2003), Corseuil et al. (2004), Iserhard \& Romanowski (2004), Quadros et al. (2004), Marchiori \& Romanowski (2006), Teston et al. (2006), Giovenardi et al. (2008), Paz et al. (2008), Iserhard et al. (2010). Foi calculada a análise de similaridade de Jaccard entre os ambientes, através do programa Past (Hammer et al. 2001).

\section{Resultados e Discussão}

Após um total de 99 horas de amostragem com rede entomológica, foram registradas 46 espécies de borboletas (Tabela 1), distribuídas em 6 famílias e 19 subfamílias para o PNMU. Ressalta-se a riqueza desta área de Pampa, e a necessidade de realização de inventários rápidos de fauna, pois com um esforço amostral em torno de um terço em relação ao vizinho Parque Estadual do Espinilho (vide Marchiori \& Romanowski 2006), foram registradas em torno de 50\% da riqueza de espécies encontrada no PEE e entorno. É importante frisar também que a gama de ambientes contemplados no estudo de Marchiori \& Romanowski (2006) foi maior em relação ao PNMU.

A proporção de espécies por família de borboleta se equipara aos dados obtidos em outros trabalhos realizados no Rio Grande do Sul, os quais diferem do restante do país. A família com maior representatividade foi Nymphalidae (50\%), seguida de Hesperiidae (24\%), Pieridae $(15,2 \%)$, Lycaenidae + Riodinidae $(8,7 \%)$ e Papilionidae $(2,1 \%)$ (Tabela 2). Dados similares foram obtidos no Parque Estadual do Espinilho, onde Nymphalidae aparece também como a família mais representativa, mas com um percentual menor (Tabela 2). Marchiori \& Romanowski (2006) destacam a diferença de proporção com a diminuição de Lycaenidae e aumento de Nymphalidae do PEE e seu entorno em relação a outros inventários realizados no país. A família Nymphalidae, seguida por Hesperiidae e Lycaenidae, foram as mais representativas no estudo de Iserhard \& Romanowski (2004) para uma área de Floresta Ombrófila Densa no Rio Grande do Sul. No presente trabalho há uma inversão de riqueza entre Lycaenidae e Pieridae, sendo a última possuidora de mais espécies em relação a Lycaenidae (Tabela 2), padrão este não evidenciado no Parque Estadual do Espinilho e na Mata Atlântica do Rio Grande do Sul. Porém, cabe ressaltar a diferença marcante na fisionomia e composição da vegetação entre a Floresta Atlântica e o Pampa, o que pode modificar a representatividade e composição das famílias de borboletas associadas a cada um desses biomas. Mesmo assim, acredita-se que com o aumento do esforço amostral, haveria uma modificação na representatividade destas famílias para o PNMU, principalmente em Lycaenidae e Hesperiidae. Tais famílias são mais difíceis de capturar devido ao seu tamanho diminuto e sua agilidade no voo, além de ser necessário um esforço maior de amostragem para que sua representatividade seja maior.

Tabela 2. Riqueza de espécies para famílias de borboletas registradas para o Parque Natural Municipal de Uruguaiana (PNMU) durante o período de julho de 2008 a maio de 2009 e para o Parque Estadual do Espinilho (PEE) durante o período de março de 2003 a janeiro de 2004. Números entre parênteses indicam porcentagem. *Família Riodinidae incluída em Lycaenidae.

Table 2. Species richness of butterfly families recorded for Parque Natural Municipal de Uruguaiana (PNMU) between July 2008 and May 2009 and for Parque Estadual do Espinilho (PEE) between March 2003 and January 2004. Values in brackets indicate percentage. *Riodinidae included in Lycaenidae.

\begin{tabular}{lcr}
\hline Famílias & PNMU & \multicolumn{1}{c}{ PEE } \\
\hline Nymphalidae & $23(50)$ & $36(37,1)$ \\
Hesperiidae & $11(24)$ & $25(25,9)$ \\
Pieridae & $7(15,2)$ & $14(14,2)$ \\
Lycaenidae* & $4(8,6)$ & $18(18,7)$ \\
Papilionidae & $1(2,2)$ & $4(4,1)$ \\
\hline
\end{tabular}

Em relação a espécies comuns entre o Parque Natural Municipal de Uruguaiana e o Parque Estadual do Espinilho, Doxocopa laurentia laurentia (Godart, 1824) foi registrada em ambas as localidades apenas em áreas de mata ciliar. Onze espécies registradas no PNMU não haviam sido listadas por Marchiori \& Romanowski (2006), com isto a riqueza de borboletas desta região do extremo sudoeste do Pampa chega a 108 espécies. Determinadas espécies aqui citadas e não relacionadas para o PEE são frequentes em áreas úmidas, clareiras de matos e moitas floridas, como, por exemplo, Carrhenes canescens pallida Röber, 1925. Representantes de Ithomiinae, Dircenna dero celtina Burmeister, 1878 e Mechanitis lysimnia lysimnia (Fabricius, 1793) ocorreram somente na mata ciliar do PNMU. Estas espécies se alimentam, nos seus estágios imaturos, de plantas da família Solanaceae, especificamente da planta Solanum mauritianum Scop. (Biezanko 1960a), a qual é frequentemente encontrada na mata ciliar do PNMU.

Ocorreram dois novos registros de borboletas para o Rio Grande do Sul, Fountainea glicerium cratais (Hewitson, 1874) e Pyrrhopyge pelota Plötz, 1879 e um registro novo para o Brasil, Tatochila mercedis vanvolxemii (Capronnier, 1874). Fountainea glicerium cratais pertence à Nymphalidae, subfamília Charaxinae, e foi registrada no verão na trilha MC. Pyrrhopyge pelota pertence à Hesperiidae, subfamília Pyrrhopyginae, apresenta como hábito buscar áreas ensolaradas, com temperaturas mais elevadas e recursos alimentares (flores) abundantes (Fonseca et al. 2006). Foi registrada na área de entorno do PNMU, trilha CS. Imaturos desta espécie se alimentam de espécies do gênero Psidium (Myrtaceae) (Biezanko 1963), planta comum na área de estudo. Tatochila mercedis vanvolxemii, representante de Pieridae, subfamília Pierinae foi registrada na primavera, na trilha CS. Apesar de Pieridae ser amplamente distribuída e bem representada no estado e no Brasil, em geral, por borboletas de hábitos generalistas e por possuírem uma riqueza de espécies menor e bem conhecida quando comparada as demais famílias de borboletas, Tatochila mercedis vanvolxemii possuía ocorrência para a Argentina (Canals 2000), sendo descrita por Bustos (2008) como uma subespécie endêmica da Argentina (com ocorrência no sul e oeste), mas podendo ser encontrada, também, no nordeste da província de Buenos Aires.

Entre as trilhas amostradas no PNMU foi possível constatar que estas apresentam uma diferença de composição relativamente alta entre si, pelos baixos valores de similaridade verificados através do Índice de Jaccard. As trilhas menos similares foram MR e CS $(0,275)$, e as mais similares foram MC e MR $(0,306)$. Tais resultados podem estar associados com a diferença na composição da vegetação em cada ambiente.

A região Sudoeste do Rio Grande do Sul, assim como toda área do estado que margeia o Rio Uruguai ainda é muito pouco representada em estudos, tanto no que tange a fauna de borboletas como de outros taxa. Provavelmente muitas outras espécies de borboletas devem ocorrer nesta região de Pampa, já que este inventário foi realizado em tempo exíguo e em relativamente poucas ocasiões amostrais. Porém, tais resultados obtidos são comparáveis a outros inventários de borboletas realizados no Rio Grande do Sul com mesma metodologia, e em certos casos indicam inclusive uma semelhança nos resultados.

Inventários de curto prazo podem revelar características importantes das comunidades locais, tais como utilização de recursos e a preferência de habitat, além de contribuir para o conhecimento da fauna regional (Marchiori \& Romanowski 2006). Desta forma, tais estudos possibilitam uma melhor compreensão do organismo em questão e sua associação com determinado ambiente visando contribuir com atividades futuras de manejo, monitoramento e conservação. 


\section{Agradecimentos}

Aos colegas e amigos do Laboratório NUPILABRU (Núcleo de Pesquisas Ictiológicas, Limnológicas e Aquicultura da Bacia do Rio Uruguai) da PUCRS e do Laboratório de Ecologia de Insetos (UFRGS). Aos familiares pelo auxílio e apoio em todas as atividades pertinentes ao desenvolvimento desta pesquisa. Ao Dr. Olaf Mielke (UFPR) pela identificação de Hesperiidae e pela revisão do trabalho, e a um revisor anônimo pelas críticas e sugestões. A Dra. Helena Piccoli Romanowski (UFRGS) pela acolhida, disponibilização de material bibliográfico e incentivo a novos pesquisadores.

\section{Referências Bibliográficas}

BIEZANKO, C.M. 1949. Acraeidae, Heliconiidae et Nymphalidae de Pelotas e seus arredores. Contribuição ao conhecimento da fisiografia do Rio Grande do Sul. Livraria Globo, Pelotas, 16p.

BIEZANKO, C.M. 1958. Ib. Pieridae da Zona Sueste do Rio Grande do Sul. Arq. Entomol., Ser. A:1-15.

BIEZANKO, C.M. 1959a. Ia. Papilionidae da Zona Sueste do Rio Grande do Sul. Arq. Entomol., Ser. A:1-17.

BIEZANKO, C.M. 1959b. Ia. Papilionidae da Zona Missioneira. Arq Entomol., Ser. B:1-12.

BIEZANKO, C.M. 1960a. Ib. Pieridae da Zona Missioneira do Rio Grande do Sul. Arq. Entomol., Ser. B:1-12.

BIEZANKO, C.M. 1960b. III. Danaidae et Ithomidae da Zona Sueste do Rio Grande do Sul. Arq. Entomol., Ser. A:1-6.

BIEZANKO, C.M. 1960c. III. Danaidae et Ithomidae da Zona Missioneira do Rio Grande do Sul. Arq. Entomol., Ser. B:1-6.

BIEZANKO, C.M. 1960d. IV. Satyridae, Morphidae et Brassolidae da Zona Sueste do Rio Grande do Sul. Arq. Entomol., Ser. A:1-13.

BIEZANKO, C.M. 1960e. IV. Satyridae, Morphidae et Brassolidae da Zona Missioneira do Rio Grande do Sul. Arq. Entomol., Ser. B:1-10.

BIEZANKO, C.M. 1963. VI. Hesperiidae da Zona Sueste do Rio Grande do Sul. Arq. Entomol., Ser. A:1-25.

BIEZANKO, C.M. \& MIELKE, O.H.H. 1973. Contribuição ao estudo faunístico dos Hesperiidae americanos. IV Espécies do Rio Grande do Sul, Brasil, com notas taxonômicas e descrições de espécies novas (Lepidoptera). Acta Biol. Parana. 2(1-4):51-102.

BORTOLUZZI, L.R. \& SOUZA, M.V. 2007. O dia do Pampa. Biodiv. Pampeana 5(2):1-2.

BROWN, K.S. \& FREITAS, A.V.L. 1999. Lepidoptera. In Biodiversidade do Estado de São Paulo, Brasil. Invertebrados Terrestres (C.R.F. Brandão \& E.M. Cancello, eds.). São Paulo, FAPESP, p. 225-245.

BUSTOS, E.N. 2008. Las especies urbanas de Rhopalocera de la Reserva Ecológica Costanera Sur, Ciudad de Buenos Aires, Argentina (Lepidoptera: Hesperioidea y Papilionoidea). SHILAP Revta. Lepid. 36(144):435-447.

CANALS, G.R. 2000. Mariposas bonaerenses. L.O.L.A., Buenos Aires, 347p. CANALS, G.R. 2003. Mariposas de misiones. L.O.L.A., Buenos Aires, 492p.

CORSEUIL, E., QUADROS, F.C., TESTON, J.A. \& MOSER, A. 2004. Borboletas (Lepidoptera: Papilionoidea e Hesperioidea) coletadas no Centro de Pesquisa e Conservação da Natureza Pró-Mata. 4: Lycaenidae. Divul. Mus. Ciênc. Tecnol. PUCRS 9:65-70.

D'ABRERA, B. 1981. Butterflies of the Neotropical Region. Part I. Papilionidae \& Pieridae. Victoria, Hill House, 72p.

EMERY, E.O., BROWN, K.S. \& PINHEIRO, C.E.G. 2006. As borboletas (Lepidoptera, Papilionoidea) do Distrito Federal, Brasil. Rev. Bras. Entomol. 50(1):85-92.

FONSECA, N.G., KUMAGAI, A.F. \& MIELKE, O.H.H. 2006. Lepidópteros visitantes florais de Stachytarpheta cayennensis (Rich.) Vahl (Verbenaceae) em remanescentes de Mata Atlântica, Minas Gerais, Brasil. Rev. Bras. Entomol. 50(3):399-405.
GIOVENARDI, R., DI MARE, R.A., SPONCHIADO, J., ROANI, S.H., JACOMASSA, F.A.F., JUNG, A.B. \& PORN, M.A. 2008. Diversidade de Lepidoptera (Papilionoidea e Hesperioidea) em dois fragmentos de floresta no município de Frederico Westphalen, Rio Grande do Sul, Brasil. Rev. Bras. Entomol. 52(4):599-605.

HAMMER, Ø., HARPER, D.A.T. \& RYAN, P.D. 2001. Paleontological statistics - PAST. Version 1.18. http://folk.uio.no/ohammer/past (último acesso em 14/07/2010).

INSTITUTO BRASILEIRO DE GEOGRAFIA E ESTATÍSTICA - IBGE. 2005. Mapas de biomas e vegetação. http://www.ibge.gov.br/home/ presidencia/noticias/noticia_visualiza.php?id_noticia=169 (último acesso em 05/08/09).

ISERHARD, C.A. \& ROMANOWSKI, H.P. 2004. Lista de espécies de borboletas (Lepidoptera, Papilionoidea e Hesperioidea) da região do Vale do rio Maquiné, Rio Grande do Sul, Brasil. Rev. Bras. Zool. 21(3):649-662.

ISERHARD, C.A., QUADROS, M.T., ROMANOWSKI, H.P. \& MENDONÇAJR, M.S. 2010. Borboletas (Lepidoptera: Papilionoidea e Hesperioidea) ocorrentes em diferentes ambientes na Floresta Ombrófila Mista e nos Campos de Cima da Serra do Rio Grande do Sul, Brasil. Biota Neotrop. 10(1):309-320: http://www.biotaneotropica.org.br/v10n1/pt/abstract?in ventory+bn02910012010.

KRÜGER, C.P. \& SILVA, E.J.E. 2003. Papilionoidea (Lepidoptera) de Pelotas e seus arredores, Rio Grande do Sul, Brasil. Entomol. Vectores 10(1):31-45.

LAMAS, G. 2004. Atlas of Tropical Lepidoptera. Checklist: Part 4a Hesperioidea - Papilionoidea. Scientific Publishers; Association for Tropical Lepidoptera, Gainesville, 439p.

LAMAS, G. 2008. La sistemática sobre mariposas (Lepidoptera: Hesperioidea y Papilionoidea) en el mundo: estado actual y perspectivas futuras. In III Reunión Anual de la Red Iberoamericana de Biogeografía y Entomología Sistemática. La Plata, Argentina. p. 57-70.

LEWINSOHN, T.M. 2006. Avaliação do estado do conhecimento da biodiversidade brasileira. Ministério do Meio Ambiente, Brasília.

MALUF, J.R.T. 2000. Nova classificação climática para o Estado do Rio Grande do Sul. Rev. Bras. Agrometereol. 8(1):141-150.

MARCHIORI, M.O. \& ROMANOWSKI, H.P. 2006. Borboletas (Lepidoptera, Papilionoidea e Hesperioidea) do Parque Estadual do Espinilho e entorno, Rio Grande do Sul, Brasil. Rev. Bras. Zool. 23(4):1029-1037.

MIELKE, O.H.H. 1980a. Contribuição ao estudo faunístico dos Hesperiidae americanos. V Nota suplementar-As espécies de Pyrrhopyginae e Pyrginae do Rio Grande do Sul, Brasil (Lepidoptera). Acta Biol. Parana. 8-9:7-17.

MIELKE, O.H.H. 1980b. Contribuição ao estudo faunístico dos Hesperiidae americanos. VI Nota suplementar - As espécies de Hesperiinae do Rio Grande do Sul, Brasil (Lepidoptera). Acta Biol. Parana. 8-9:127-172.

MIELKE, O.H.H. 2005. Catalogue of the American Hesperioidea: Hesperiidae (Lepidoptera). Sociedade Brasileira de Zoologia, Curitiba, 6v., 1536p.

MORAIS, A.B.B., ROMANOWSKI, H.P., ISERHARD, C.A., MARCHIORI, M.O. \& SEGUI, R. 2007. Mariposas del Sur de Sudamérica (Lepidoptera: Papilionoidea e Hesperioidea). Ciênc. Ambient. 35:29-46.

OVERBECK, G.E., MÜLLER, S.C., FIDELIS, S., PFADENHAUER, J., PILLAR, V.P., BRANCO, C.C., BOLDRINI, I.I., BOTH, R. \& Forneck, E. 2007. Brasil's neglected biome: the South Brazilian Campos. Perspectives in Plant Ecology, Evolution and Systematics 9:101-116.

PAZ, A.L.G., ROMANOWSKI, H.P. \& MORAIS, A.B.B. 2008. Nymphalidae, Papilionidae e Pieridae (Lepidoptera: Papilionoidea) da Serra do Sudeste do Rio Grande do Sul, Brasil. Biota Neotrop. 8 (1):22-29: http://www. biotaneotropica.org.br/v8n1/pt/abstract?inventory+bn01608012008.

PILLAR, V.P., MÜLLER, S.C., CASTILHOS, Z.M.S. \& JACQUES, A.V.A. 2009. Campos Sulinos - conservação e uso sustentável da biodiversidade. Ministério do Meio Ambiente, Brasília, 409p.

QUADROS, F.C., DORNELES, A.L. \& CORSEUIL, E. 2004. Ninfalídeos (Lepidoptera, Nymphalidae) ocorrentes no norte da Planície Costeira do Rio Grande do Sul. Biociências 12(2):147-164.

RADAMBRASIL. 1986. Geologia, geomorfologia, pedologia, vegetação, uso potencial da Terra. Levantamento de recursos Naturais. IBGE, Rio de Janeiro. 
ROMANOWSKI, H.P., ISERHARD, C.A. \& HARTZ, S.M. 2009. Borboletas da floresta com araucária. In Floresta de araucária: ecologia, conservação e desenvolvimento sustentável (C.R. Fonseca, A.F. Souza, A.M. LealZanchet, T. Dutra, A. Backes \& G. Ganade, eds.). Holos Editora, Ribeirão Preto, p. 229-240.

TESTON, J.A. \& CORSEUIL, E. 1998. Lista documentada dos Papilionídeos (Lepidoptera, Papilionidae) do Rio Grande do Sul, Brasil. Biociências 6(2):81-94.

TESTON, J.A. \& CORSEUIL, E. 2000. Lista documentada dos Pierídeos (Lepidoptera, Pieridae) do Rio Grande do Sul, Brasil. Biociências $8(2): 115-132$.

TESTON, J.A. \& CORSEUIL, E. 2001. Ninfalídeos (Lepidoptera, Nymphalidae) ocorrentes no Rio Grande do Sul, Brasil. Parte I. Danainae e Ithomiinae. Biociências 9(1):51-61.
TESTON, J.A. \& CORSEUIL, E. 2008a. Ninfalídeos (Lepidoptera, Nymphalidae) ocorrentes no Rio Grande do Sul, Brasil. Parte IV. Apaturinae e Charaxinae. Biociências 16(1):28-32.

TESTON, J.A. \& CORSEUIL, E. 2008b. Ninfalídeos (Lepidoptera, Nymphalidae) ocorrentes no Rio Grande do Sul, Brasil. Parte V. Biblidinae e Limenitidinae. Biociências 16(1):33-41.

TESTON, J.A. \& CORSEUIL, E. 2008c. Ninfalídeos (Lepidoptera, Nymphalidae) ocorrentes no Rio Grande do Sul, Brasil. Parte VI. Nymphalinae e Satyrinae. Biociências 16(1):42-51.

TESTON, J.A., TOLEDO, K.G. \& CORSEUIL, E. 2006. Ninfalídeos (Lepidoptera, Nymphalidae) ocorrentes no Rio Grande do Sul, Brasil. Parte III. Heliconiinae e Libytheinae. Biociências 4(2):208-213.

WEYMER, G. 1894. Exotische Lepidopteren. VII. Beitrag zur Lepidopterenfauna von Rio Grande do Sul. Stett. Entomol. Zeitung 55(10-12):311-333.

Recebido em 10/08/2010

Versão reformulada recebida em 27/11/2010

Publicado em 01/01/2011 